\title{
Adaptive Neural Control for Hysteresis Motor Driving Servo System with Bouc-Wen Model
}

\author{
Xuehui Gao \\ Department of Mechanical and Electrical Engineering, Shandong University of Science and Technology, Tai'an 271019, China \\ Correspondence should be addressed to Xuehui Gao; xhgao@163.com
}

Received 21 April 2018; Revised 24 May 2018; Accepted 20 June 2018; Published 26 July 2018

Academic Editor: Wenbo Wang

Copyright ( 2018 Xuehui Gao. This is an open access article distributed under the Creative Commons Attribution License, which permits unrestricted use, distribution, and reproduction in any medium, provided the original work is properly cited.

\begin{abstract}
An adaptive high-order neural network (HONN) control strategy is proposed for a hysteresis motor driving servo system with the Bouc-Wen model. To simplify control design, the model is rewritten as a canonical state space form firstly through coordinate transformation. Then, a high-gain state observer (HGSO) is proposed to estimate the unknown transformed state. Afterward, a filter for the tracking errors is adopted which converts the vector error $e$ into a scalar error $s$. Finally, an adaptive HONN controller is presented, and a Lyapunov function candidate guarantees that all the closed-loop signals are uniformly ultimately bounded (UUB). Simulations verified the effectiveness of the proposed neural network adaptive control strategy for the hysteresis servo motor system.
\end{abstract}

\section{Introduction}

The servo motor system has been widely utilized in industry and military (vehicle systems [1-3], robotic systems [4-7], radar systems [8-10], and nonlinear turn table systems [11-15]). But hysteresis nonlinearity exists in these motor driving servo systems. The existence of the hysteresis can increase the control difficulty and decrease the controller performance; even more seriously, it can damage the stability of the motor servo system. Since the accuracy requirement becomes more and more strict today, hysteresis nonlinearity must be dealt with under a higher precision in these motor servo systems.

Hysteresis nonlinearity is a classic nonlinearity, and it has been investigated for many years. Plenty of results are obtained, and the hysteresis model can be roughly divided into two classes: the physical-based model and the mathematicalbased model. The typical hysteresis physical-based model is the Jiles-Atherton (J-A) model [16-18]. The physical model is constructed with the physical parameters of the hysteresis systems. Different from the physical-based model, the mathematical-based model has no connection with real physical parameters, and it is described by mathematical arguments. The mathematical-based model includes the
Preisach model [19-21], Bouc-Wen model [22-24], and Prandtl-Ishlinskii (P-I) model [25-27].

The Preisach model is the earliest mathematical-based model and has been used for system identification and control. Gao et al. [19] proposed new identification approaches and a controller for an unknown-order Hammerstein system where hysteresis nonlinearity was modeled through the Preisach model. The Hankel matrix and blind identification estimated the order and the linear section. Afterward, a new triangle matrix approach was proposed to identify the discrete Preisach model. Finally, a composite controller was designed for the hysteresis system. In [20], by utilizing the so-called Preisach plane, the Preisach model was reexpressed into a control-oriented form, in which the input signal was explicitly expressed. And a prescribed adaptive control approach was adopted to ensure the performance.

The P-I model is another popular hysteresis mathematical-based model. Many researchers focus on the P-I model investigation. Zhang et al. [26] proposed a fuzzy approximator for unknown time-delay asymmetric hysteresis nonlinear systems with a shifted Prandtl-Ishlinskii (ASPI) model through an adaptive dynamic surface control scheme, and the Krasovskii functionals have been adopted without 
the inverse ASPI model. An asymmetric hysteresis P-I model was presented in [27] for piezoelectric actuators, and two different hybrid controllers were compared to verify the control strategy where the proposed approach had better tracking precision.

The Bouc-Wen model was firstly proposed by Bouc in 1971 [28]. In 1976, Wen [29] expanded the model into a mathematical model. In recent years, the Bouc-Wen model has attracted more and more researchers' interest. Ahmad [22] proposed a new robust controller with two degrees of freedom for a Bouc-Wen hysteresis system. The proposed controller had better performance and robustness. In [23], a Bouc-Wen model was identified by the bat-inspired algorithm for a piezoactuated hysteresis system. A sliding mode control method was designed suppressing the hysteresis nonlinearity. Comparing with another two mathematical-based hysteresis models, the Bouc-Wen model has more parameters, but it only needs differential calculation. Then, it is easier to implement. Thus, the Bouc-Wen hysteresis model should attract more attention for hysteresis investigations.

For nonlinear systems, the HONN has been focused on for many years [30-32]. Ge et al. [30] investigated a HONN-adaptive control strategy for the MIMO systems. A HONN controller with adaptive laws was designed, and the tracking errors were convergent to an adjustable compact set. In [33], a HONN control was designed by the backstepping. However, to the author's knowledge, HONN is rarely utilized to approximate the Bouc-Wen hysteresis nonlinearity for control systems.

This paper investigates an adaptive HONN control for hysteresis nonlinearity motor driving servo system with the Bouc-Wen model. The system with hysteresis is first reformulated as a canonical state space form, where the unknown dynamics are all lumped together to simplify the control design. In order to estimate the unknown transformed states, a HGSO is proposed and then the adaptive HONN controller is designed. Next, to simplify the controller design, a filter will be adopted to transform the tracking error into a scalar error $s$, and a Lyapunov function candidate guarantees all the signals bounded in the closed-loop system. Finally, simulation results will demonstrate the effectiveness of the HONN-adaptive controller for hysteresis nonlinearity with the Bouc-Wen model of the motor driving servo system.

\section{Problem Formulation}

2.1. Hysteresis Servo Motor Driving System with the BoucWen Model. Based on the literature [34], considering the hysteresis servo motor driving system as

$$
\begin{aligned}
J \ddot{\theta}+b \dot{\theta} & =u-\tau, \\
J_{m} \ddot{\theta}_{m}+f_{m}\left(\dot{\theta}_{m}\right) & =\tau,
\end{aligned}
$$

where $\theta$ and $\theta_{m}$ describe angle positions of the motor and load, respectively; $\dot{\theta}$ and $\dot{\theta}_{m}$ are their velocities, respectively; $J$ and $J_{m}$ represent the moments of inertia of the motor and load, respectively; $b$ is the viscous friction coefficient of the

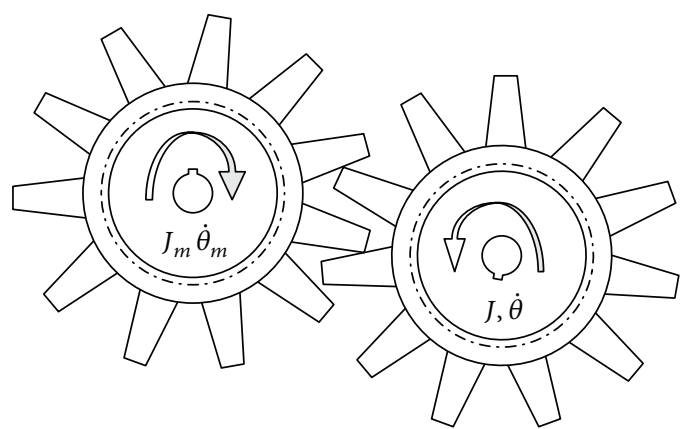

Figure 1: The hysteresis servo motor system structure.

TABle 1: The Bouc-Wen model parameters.

\begin{tabular}{lcccccc}
\hline Hysteresis parameters & $\mu_{1}$ & $\mu_{2}$ & $\alpha$ & $\beta$ & $n$ & $\zeta\left(t_{0}\right)$ \\
\hline Positive direction & 3 & 5 & 3.2 & 0.5 & 2 & 0 \\
Negative direction & -3 & -5 & 3.2 & 0.5 & 2 & 0 \\
\hline
\end{tabular}

motor; $u$ is the input torque of the motor; $f_{m}$ is the friction torque of the load; and $\tau$ represents transmission torque, which can be formulated with the Bouc-Wen hysteresis model as

$$
\begin{aligned}
\tau & =\mu_{1} u(t)+\mu_{2} \zeta(t), \\
\dot{\zeta}(t) & =\dot{u}(t)-\alpha|\dot{u}(t) \| \zeta(t)|^{n-1} \zeta(t)-\beta \dot{u}(t)|\zeta(t)|^{n} \\
& =\dot{u}(t)-F(\dot{u}(t), \zeta(t)),
\end{aligned}
$$

where sign $\left(\mu_{1}\right)=\operatorname{sign}\left(\mu_{2}\right), \zeta\left(t_{0}\right)=0, \alpha>|\beta|$, and $F(\dot{u}(t)$, $\zeta(t))=\alpha|\dot{u}(t) \| \zeta(t)|^{n-1} \zeta(t)+\beta \dot{u}(t)|\zeta(t)|^{n}$. The structure of the hysteresis servo motor system is illustrated in Figure 1.

In the Bouc-Wen model, the parameter $\beta$ decides the hysteresis shape and hysteresis amplitude, the parameter $n$ denotes the smoothness of the slope, and $\mu_{1}$ means the direction of the hysteresis nonlinearity. If the input is chosen as $u(t)=4 \sin (2 \pi t)$, the Bouc-Wen parameters are selected in Table 1. Then, the images of the Bouc-Wen model are illustrated by Figures 2(a) and 2(b).

2.2. Hysteresis Servo Motor Driving State Space Model. The hysteresis servo motor driving system (1) will be rewritten as a state space status to facilitate the controller design. The state space variables can be defined as

$$
\begin{aligned}
& z_{1}=\theta_{m}, \\
& z_{2}=\dot{\theta}_{m}, \\
& z_{3}=\theta, \\
& z_{4}=\dot{\theta} .
\end{aligned}
$$

Then, the hysteresis servo motor driving system (1) is rewritten as 


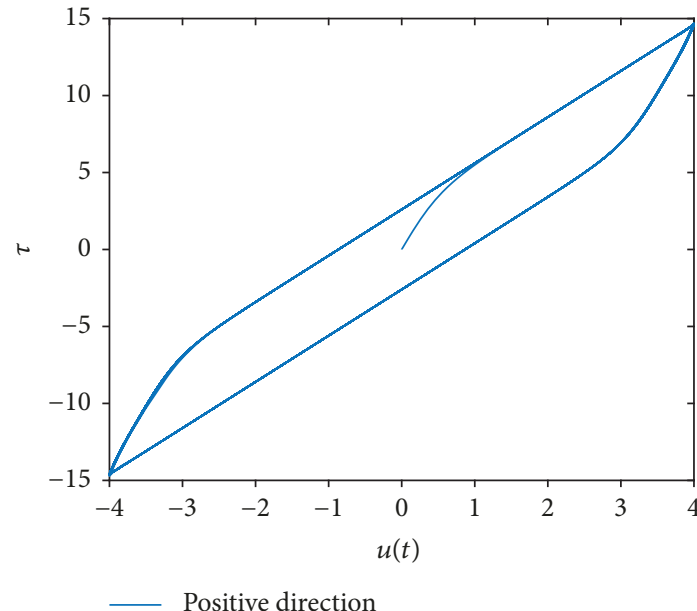

(a) The Bouc-Wen positive curve

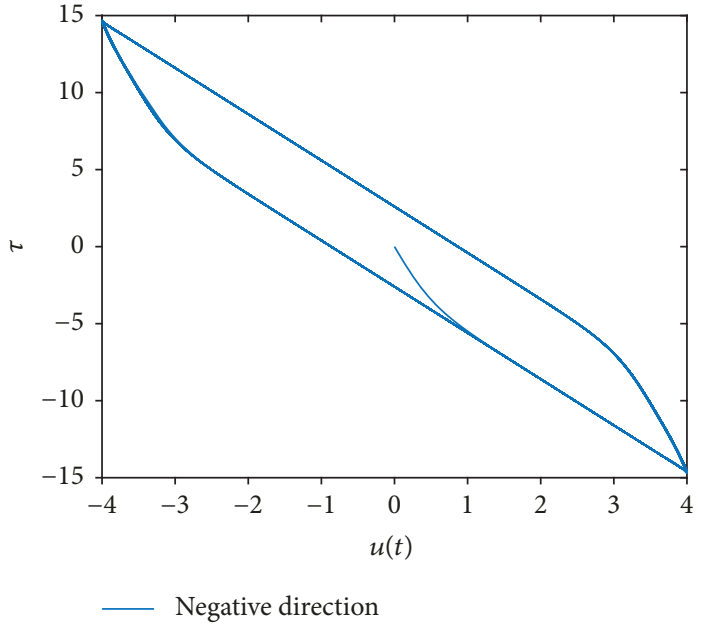

(b) The Bouc-Wen negative curve

Figure 2: The curve of the Bouc-Wen model.

$$
\begin{aligned}
& \dot{z}_{1}=z_{2}, \\
& \dot{z}_{2}=a_{1}\left(\mu_{1} u+\mu_{2} \zeta\right)-a_{1} f_{m}\left(z_{2}\right), \\
& \dot{z}_{3}=z_{4}, \\
& \dot{z}_{4}=a_{2} u\left(1-\mu_{1}-\mu_{2} \zeta\right)+a_{3} z_{4},
\end{aligned}
$$

where $a_{1}=1 / J_{m}, a_{2}=1 / J$, and $a_{3}=b / J$.

Considering that $z_{1}=\theta_{m}$ represents angle positions of load, which is the output of the nonlinear system, we, therefore, have $y=z_{1}$.

In order to simplify the design of the control strategy, the state space model (4) should be rewritten in a pure-feedback form. In view of reference [35], $\mathrm{Na}$ et al. proposed a new coordinate transformation to transform the pure-feedback system into a canonical system. Similar to the approach of [35], we also adopted a coordinate transformation to transform (4) into a canonical state space form.

Define the alternative state variables as

$$
\begin{aligned}
& x_{1}=z_{1}, \\
& x_{2}=\dot{x}_{1}, \\
& x_{3}=\dot{x}_{2}, \\
& x_{4}=\dot{x}_{3}, \\
& y=x_{1}=z_{1} .
\end{aligned}
$$

According (4) and (5), it has $x_{2}=\dot{x}_{1}=z_{2}$; then, the follow equation can be deduced as

$$
\dot{x}_{2}=\ddot{x}_{1}=\frac{\partial z_{2}}{\partial x_{1}} \dot{x}_{1}+\frac{\partial z_{2}}{\partial x_{2}} \dot{x}_{2}=\bar{a}_{2}\left(z_{1}, z_{2}\right)+\bar{b}_{2}\left(z_{1}, z_{2}, z_{3}\right) z_{3}
$$

As an analogy, the system (4) is obtained as

$$
\begin{aligned}
& \dot{x}_{1}=x_{2}, \\
& \dot{x}_{2}=x_{3}, \\
& \dot{x}_{3}=x_{4}, \\
& \dot{x}_{4}=\bar{a} x+\bar{b}(x, u) u, \\
& y=x_{1},
\end{aligned}
$$

where $x=\left[x_{1}, x_{2}, x_{3}, x_{4}\right]^{T}$.

\section{High-Gain State Observer Design}

Considering (7), the high-gain state observer (HGSO) is proposed as

$$
\begin{aligned}
& \dot{\hat{x}}_{1}=\widehat{x}_{2}+l_{1}\left(y-\widehat{x}_{1}\right), \\
& \dot{\hat{x}}_{2}=\widehat{x}_{3}+l_{2}\left(y-\widehat{x}_{1}\right), \\
& \dot{\hat{x}}_{3}=\widehat{x}_{4}+l_{3}\left(y-\widehat{x}_{1}\right), \\
& \dot{\hat{x}}_{4}=\widehat{a} \widehat{x}+\widehat{b}(\widehat{x}, u) u+l_{4}\left(y-\widehat{x}_{1}\right), \\
& \widehat{y}=\widehat{x}_{1},
\end{aligned}
$$

where $l_{i}, i=1,2,3,4$ are the designed parameters; $\hat{x}=$ $\left[\widehat{x}_{1}, \widehat{x}_{2}, \widehat{x}_{3}, \widehat{x}_{4}\right]^{T}$ is the estimation of $x$, and $\widehat{a}$ and $\widehat{b}$ are the estimation of $\bar{a}$ and $\bar{b}$, respectively. Based on the HGSO, the following Lemma is hold.

Lemma 1. Considering the servo motor system (4), it transformed into the canonical state space form (7) and then define the HGSO as (8); then, the HGSO can approximate the system with designed $l$.

Proof 1. Define $\tilde{x}_{i}=x_{i}-\widehat{x}_{i}, \tilde{y}=y-\widehat{y}, \tilde{a}=\bar{a}-\widehat{a}, \tilde{b}=\bar{b}-\widehat{b}$. When (7) subtracts (8), one has 


$$
\begin{aligned}
& \dot{\tilde{x}}_{1}=\tilde{x}_{2}-l_{1} \tilde{x}_{1}, \\
& \dot{\tilde{x}}_{2}=\tilde{x}_{3}-l_{2} \tilde{x}_{1}, \\
& \dot{\tilde{x}}_{3}=\tilde{x}_{4}-l_{3} \tilde{x}_{1}, \\
& \dot{\tilde{x}}_{4}=\tilde{a} \tilde{x}+\tilde{b}(\tilde{x}, u) u+l_{4} \tilde{x}_{1}, \\
& \tilde{y}=\tilde{x}_{1} .
\end{aligned}
$$

Rewriting (9) as

$$
\begin{aligned}
& \dot{\tilde{x}}=A \tilde{x}+B u, \\
& \tilde{y}=\tilde{x}_{1},
\end{aligned}
$$

where

$$
\begin{aligned}
A & =\left[\begin{array}{cccc}
-l_{1} & 1 & 0 & 0 \\
-l_{2} & 0 & 1 & 0 \\
-l_{3} & 0 & 0 & 1 \\
-l_{4}+\tilde{a}_{1} & \tilde{a}_{2} & \tilde{a}_{3} & a_{4}
\end{array}\right], \\
B & =\left[\begin{array}{c}
0 \\
0 \\
0 \\
\tilde{b}(\tilde{x}, u)
\end{array}\right],
\end{aligned}
$$

and all designed parameters $l_{i}$ should be properly chosen so that the polynomial $m(z)=p^{4}+l_{1} p^{3}+l_{2} p^{2}+l_{3} p+l_{4}$ is Hurwitz. Thus, for the matrix $A$, select a positive definite symmetric matrix $P=P^{T}>0$; there exists a positive definite symmetric matrix $Q=Q^{T}>0$ such that the following inequality holds:

$$
A^{T} P+P A \leq-Q .
$$

Select the Lyapunov candidate $V_{0}$ for the state observer estimate errors as follows:

$$
V_{0}=\frac{1}{2} \tilde{x}^{T} P \tilde{x}
$$

Considering (10), (11), and (12), the derivative of $V_{0}$ is deduced as

$$
\dot{V}_{0}=\frac{1}{2} \dot{\tilde{x}}^{T} P \tilde{x} \leq-\frac{1}{2} \tilde{x}^{T} Q \tilde{x}
$$

Then, based on the method of reference [36], we have

$$
\dot{V}_{0} \leq-\rho_{1} V_{0}+\rho_{2}
$$

where $\rho_{1}>0$ and $\rho_{2}>0$. Therefore, the proposed highgain state observer can estimate the unknown states of the hysteresis system.

\section{Controller Design}

Considering the transformed motor servo system, in order to estimate the unknown parameters, a new HONN will be designed for the servo motor system. Moreover, an adaptive controller will be presented based on the new HONN, and a Lyapunov function candidate will guarantee all the signals bounded in the closed-loop of the hysteresis system.

4.1. HONN. In this section, a new HONN will be designed through the states $x=\left[x_{1}, x_{2}, x_{3}, x_{4}\right]^{T}$. To account for unknown friction $f_{m}\left(x_{2}\right)$ and unknown torque $\tau$, a $\mathrm{NN}$ approximation [35] is adopted over a compact set $\Omega$ as

$$
Q(x)=W^{* T} \Phi(x)+\varepsilon \quad \forall x \in \Omega \subset \mathbb{R}^{n},
$$

where $W^{*}=\left[\omega_{1}^{*}, \omega_{2}^{*}, \ldots, \omega_{L}^{*}\right]^{T} \in \mathbb{R}^{L}$ are the bounded $\mathrm{NN}$ weights; $\varepsilon \in \mathbb{R}$ is a bounded error, that is, $\left\|W^{*}\right\| \leq W_{N}$ and $|\varepsilon| \leq \varepsilon_{N}$ where $W_{N}$ and $\varepsilon_{N}$ are positive constants; and $\Phi(x)=\left[\Phi_{1}(x), \Phi_{2}(x), \ldots, \Phi_{L}(x)\right]^{T} \in \mathbb{R}^{L}$ is the NN base vector. High-order functions $\Phi_{j k}(x)=\prod_{j \in J_{k}}\left[\sigma\left(x_{j}\right)\right]^{d_{k}(j)}, k=1,2$, $\ldots, L$ are used, where $J_{k}$ are $L$-not-ordered subsets of $\{1,2$, $\ldots, n\}, d_{k}(j)$ are nonnegative integers, and $\sigma(\cdot)$ equals to $\sigma(x)=\kappa /\left(1+e^{-g x}\right)+q$, where $g$ and $\kappa$ are positive bounded parameters and the real number $q$ is also bound.

4.2. Tracking Error Definition. In this section, we define a reference input as $y_{d}=\left[y_{d}, \dot{y}_{d}, \ldots, y_{d}^{(n-1)}\right]^{T} \in \mathbb{R}^{n}$ for the HONN-adaptive controller design, and the tracking error is selected as

$$
\begin{aligned}
& e=x-y_{d}, \\
& s=\left[\Lambda^{T} 1\right] e,
\end{aligned}
$$

where $\Lambda=\left[\Lambda_{1}, \Lambda_{2}, \ldots, \Lambda_{n-1}\right]^{T}$ is a filter vector and $s^{n-1}+$ $\Lambda_{n-1} s^{n-2}+\cdots+\Lambda_{1}$ is Hurwitz. Therefore, $e$ is bounded when $s$ is bounded.

The estimation of states is defined as $\hat{x}=\left[\widehat{x}_{1}, \hat{x}_{2}, \widehat{x}_{3}, \hat{x}_{4}\right]^{T}$. Then, we have

$$
\begin{aligned}
& \widehat{e}=\widehat{x}-y_{d}, \\
& \widehat{s}=\left[\Lambda^{T} 1\right] \widehat{e},
\end{aligned}
$$

where the reference $y_{d}$ is bounded, that is, $\left\|y_{d}\right\| \leq r_{d}$. Then, the errors $\tilde{e}$ and $\tilde{s}$ are denoted as

$$
\begin{aligned}
& \tilde{e}=e-\widehat{e}=x-\widehat{x}=\tilde{x}, \\
& \tilde{s}=s-\widehat{s}=\left[\Lambda^{T} 1\right] \tilde{x} .
\end{aligned}
$$

Substituting (4) into (17), we have

$$
\begin{aligned}
\dot{s}= & {\left[0 \Lambda^{T}\right] e+\left(W_{1}^{* T} \Phi_{1}\left(x_{2}, x_{3}\right)+\varepsilon_{1}\right) x_{3} } \\
& +a_{2} u\left(1-\mu_{1}-\mu_{2} \zeta\right)+a_{3} x_{4}-y_{d}^{(4)} .
\end{aligned}
$$

4.3. Adaptive NN Controller Design. For the hysteresis servo motor system (4), the designed HONN control is adopted as

$$
u=\frac{1}{a_{2}\left(1-\mu_{1}\right)}\left(-k \widehat{s}+\widehat{W} \Phi(x)+y_{d}^{(4)}+a_{2} \mu_{2} \zeta\right)
$$

where $k>0$ and $\widehat{W}$ is the estimated weights.

The HONN weights are defined as 


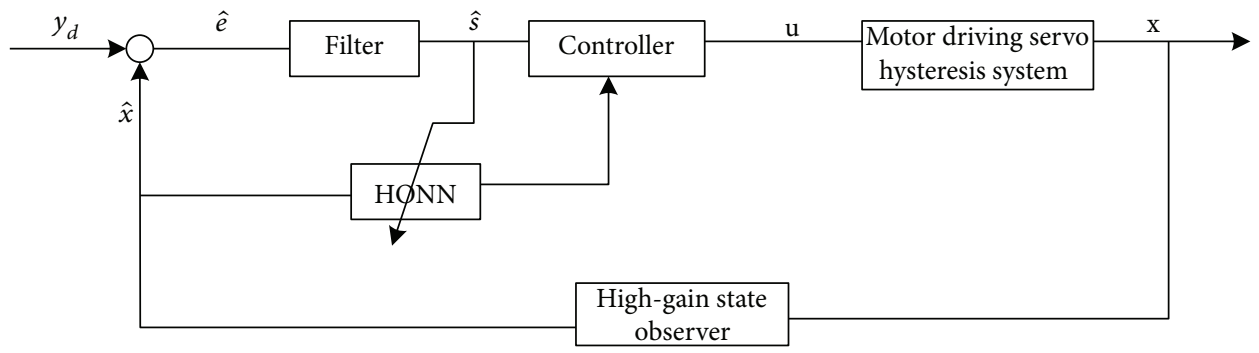

Figure 3: The control structure.

$$
\dot{\widehat{W}}=\varphi \widehat{s} \Phi^{T}(x)-\omega|\widehat{s}| \widehat{W},
$$

where the adjustment coefficients $\varphi>0$ and $\omega>0$ are the designed parameters.

Then, the Lemma is held.

Lemma 2 [35]. The $\widehat{W}$ in (22) are bounded with $\|\widehat{W}\| \leq c_{\phi} / \omega$, where $c_{\phi}$ is bounded, that is, $\|\Phi\| \leq c_{\phi}$. If the error $\tilde{W}$ is defined as $\tilde{W}=W^{*}-\widehat{W}$, then it has $\|\tilde{W}\| \leq c_{W}$, where $c_{W}=W_{N}+$ $\left(c_{\phi} / \varpi\right)$ and $W_{N}$ is a positive constant which suits $\left\|W^{*}\right\| \leq W_{N}$.

We define $F_{1}\left(\widehat{e}, x_{3}\right)=\left[0 \Lambda^{T}\right] \widehat{e}+\left(W_{1}^{* T} \Phi_{1}\left(x_{2}, x_{3}\right)+\varepsilon_{1}\right) x_{3}$; then, it has $F_{1}\left(\widehat{e}, x_{3}\right)=W^{* T} \Phi(x)+\varepsilon$. The update law of $\tilde{x}$ is defined as

$$
\dot{\tilde{x}}_{4}=-a_{3} s .
$$

The control structure diagram is shown in Figure 3.

4.4. Stability Analysis. From Lemma 2, considering the designed HONN, the adaptive law (23), and the HONN weights (22), the following theorem is held.

Theorem 1. Considering the hysteresis servo motor system (4), the controller (21) with adaptive law (23), the HONN (16) with update law (22), all the signals in a closed loop, therefore, are UUB and the tracking errors are convergent to a small compact set as $|s| \leq \sqrt{2\left((\varsigma / \rho)+V(0) e^{-\rho t}\right)}$ with $V$ defined in (25) and $\varsigma$ and $\rho$ defined in (30).

Proof 1. The derivative of the $s$ is deduced from (19), (20), and (21) as

$$
\begin{aligned}
\dot{s}= & {\left[0 \Lambda^{T}\right](\widehat{e}+\tilde{x})+a_{2} u\left(1-\mu_{1}-\mu_{2} \zeta\right) } \\
& +\left(W_{1}^{* T} \Phi_{1}\left(x_{2}, x_{3}\right)+\varepsilon_{1}\right) x_{3}+a_{3} x_{4}-y_{d}^{(4)} \\
= & {\left[0 \Lambda^{T}\right] \widehat{e}+\left[0 \Lambda^{T}\right] \tilde{x}+a_{2} u\left(1-\mu_{1}-\mu_{2} \zeta\right) } \\
& +\left(W_{1}^{* T} \Phi_{1}\left(x_{2}, x_{3}\right)+\varepsilon_{1}\right) x_{3}+a_{3} x_{4}-y_{d}^{(4)} \\
= & F_{1}\left(\widehat{e}, x_{3}\right)+\left[0 \Lambda^{T}\right] \tilde{x}-k \widehat{s}+\widehat{W}^{T} \Phi(x) \\
& -a_{3} \widehat{x}_{4}+y_{d}^{(4)}+a_{2} \mu_{2} \zeta-a_{2} \mu_{2} \zeta+a_{3} x_{4}-y_{d}^{(4)} \\
= & W^{* T} \Phi(x)+\left[0 \Lambda^{T}\right] \tilde{x}-k \widehat{s}+\widehat{W}^{T} \Phi(x)+a_{3} \tilde{x}_{4} \\
= & \tilde{W}^{T} \Phi(x)+\varepsilon+\left[0 \Lambda^{T}\right] \tilde{x}-k \widehat{s}+a_{3} \tilde{x}_{4} .
\end{aligned}
$$

The selected Lyapunov function candidate is

$$
V=\frac{1}{2}\left(s^{2}+\tilde{x}_{4}^{2}\right)
$$

The derivative of $V$ can be deduced by (19) and (24):

$$
\dot{V}=s\left(\tilde{W}^{T} \Phi(x)+\varepsilon+\left[0 \Lambda^{T}\right] \tilde{x}-k \widehat{s}+a_{3} \tilde{x}_{4}\right)+\tilde{x}_{4} \dot{\tilde{x}}_{4} .
$$

Substituting (19) and (23) into (26), one has

$$
\begin{aligned}
\dot{V} & =s\left(-k s+k \tilde{s}+\tilde{W}^{T} \Phi(x)+\varepsilon+\left[0 \Lambda^{T}\right] \tilde{x}+a_{3} \tilde{x}_{4}\right)+\tilde{x}_{4} \dot{\tilde{x}}_{4} \\
& =-k s^{2}+k s \tilde{s}+s \tilde{W}^{T} \Phi(x)+s \varepsilon+s\left[0 \Lambda^{T}\right] \tilde{x} .
\end{aligned}
$$

In view of the method of reference [35], considering Lemma 2, substituting (19) into (27), the derivative of $V$ in (27) can be deduced as

$$
\dot{V} \leq-k s^{2}+|s| c_{W} c_{\phi}+\varepsilon_{N}+k|s|\left|\left[\Lambda^{T} 1\right] \tilde{x}\right|+|s|\left|\left[0 \Lambda^{T}\right] \tilde{x}\right| .
$$

Since $y_{d}$ is bounded, considering (18) and (19), the error $\tilde{x}$ is bounded, that is, $\|x-\hat{x}\| \leq \chi$, where $\chi$ is a positive constant. Then, we define $\rho_{1}=\left|\left[\Lambda^{T} 1\right] \tilde{x}\right| \leq \max \left\{\Lambda_{i}, 1\right\}$ $\chi$ and $\rho_{2}=\left|\left[0 \Lambda^{T}\right] \tilde{x}\right| \leq \max \left\{\Lambda_{i}\right\} \chi$ as small positive constants. It follows

$$
\dot{V} \leq-k s^{2}+|s| c_{W} c_{\phi}+\varepsilon_{N}+k \varrho_{1}|s|+\varrho_{2}|s|=-k s^{2}+k_{w}|s|+\varepsilon_{N},
$$

where $k_{w}=c_{W} c_{\phi}+k \varrho_{1}+\varrho_{2}$.

By applying Young's inequality $a b \leq\left(a^{2}+b^{2}\right) / 2$ on the term $k_{m}|s|$, the following inequation can be deduced as

$$
\dot{V} \leq-\rho V+\varsigma,
$$

where $\varsigma=\varepsilon_{N}+k \tilde{x}_{4}{ }^{2}+k_{m}^{2} / 2$ and $\rho=2 k-1$ are guaranteed positive. According to the Lyapunov theorem, the tracking error $s$ is bounded then $V$ is UUB.

Integrating both sides of (30) over $[0, T]$, we have

$$
V \leq V(0) e^{-\rho t}+\frac{\varsigma}{\rho}\left(1-e^{-\rho t}\right) \leq \frac{\varsigma}{\rho}+V(0) e^{-\rho t} .
$$

Then, considering (25), it follows that

$$
|s| \leq \sqrt{2\left(\frac{\varsigma}{\rho}+V(0) e^{-\rho t}\right)} .
$$

So that $\lim _{t \rightarrow \infty}|s| \leq \lim _{t \rightarrow \infty} \sqrt{2\left((\varsigma / \rho)+V(0) e^{-\rho t}\right)}=$ 
TABLE 2: The system physical parameters.

\begin{tabular}{lc}
\hline$J$ & $0.16 \mathrm{~kg} \mathrm{~m}^{2}$ \\
$J_{m}$ & $0.0736 \mathrm{~kg} \mathrm{~m}^{2}$ \\
$b$ & $1.47 \mathrm{Nm} \mathrm{s} / \mathrm{rad}$ \\
$\dot{\theta}$ & $1.52 \mathrm{rad} / \mathrm{s}$ \\
\hline
\end{tabular}

TABLE 3: The parameters of the Bouc-Wen hysteresis model.

\begin{tabular}{lc}
\hline$\mu_{1}$ & 1.148 \\
$\mu_{2}$ & 2.191 \\
$\alpha$ & 3.213 \\
$\beta$ & 0.114 \\
$n$ & 2 \\
$\zeta\left(t_{0}\right)$ & 0 \\
\hline
\end{tabular}
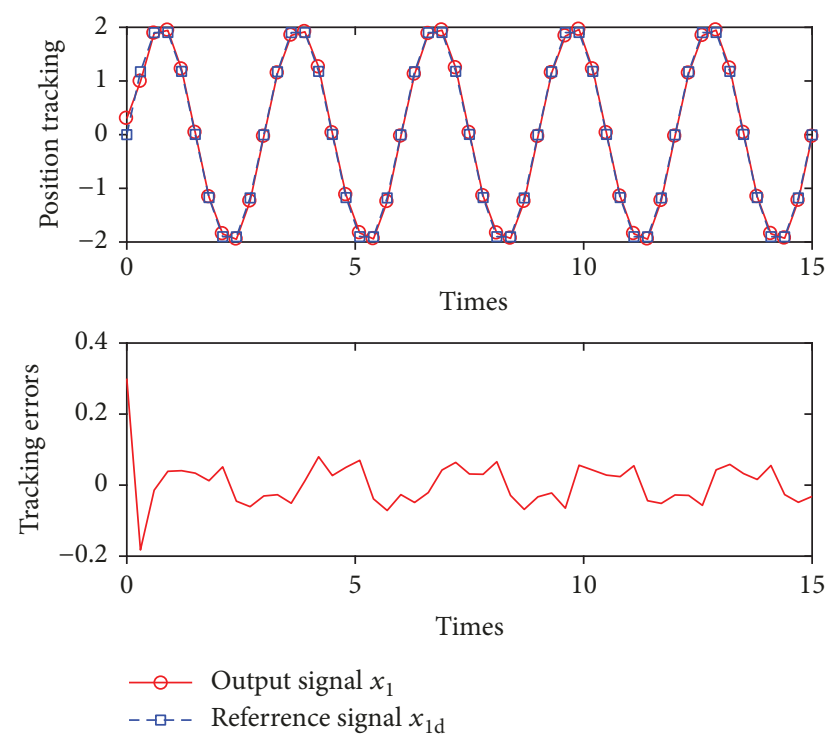

FIGURE 4: The control results and tracking error of $x_{1}$ with $y_{d}=2$ $\sin ((2 / 3) \pi t)$.

$\sqrt{2(\varsigma / \rho)}$ holds then $s$ is convergent to a small compact set. Therefore, all the signals in the closed loop are bounded.

\section{Simulations}

In this section, the proposed controller in a hysteresis servo motor system will be verified by simulations. The system parameters are shown in Table 2 . To verify the applicability of the neural adaptive controller, reference sinusoidal signals $y_{d}=2 \sin ((2 / 3) \pi t)$ and $y_{d}=2.8 \sin ((2 / 3) \pi t)$ are employed and the parameters of the Bouc-Wen model are listed in Table 3.

In the simulation, the tracking error is defined in (18) and $\Lambda$ is adopted as $\Lambda=[1,5,6]^{T}$. The controller (21) with adaptive law (23) and HONN update law (22) are implemented
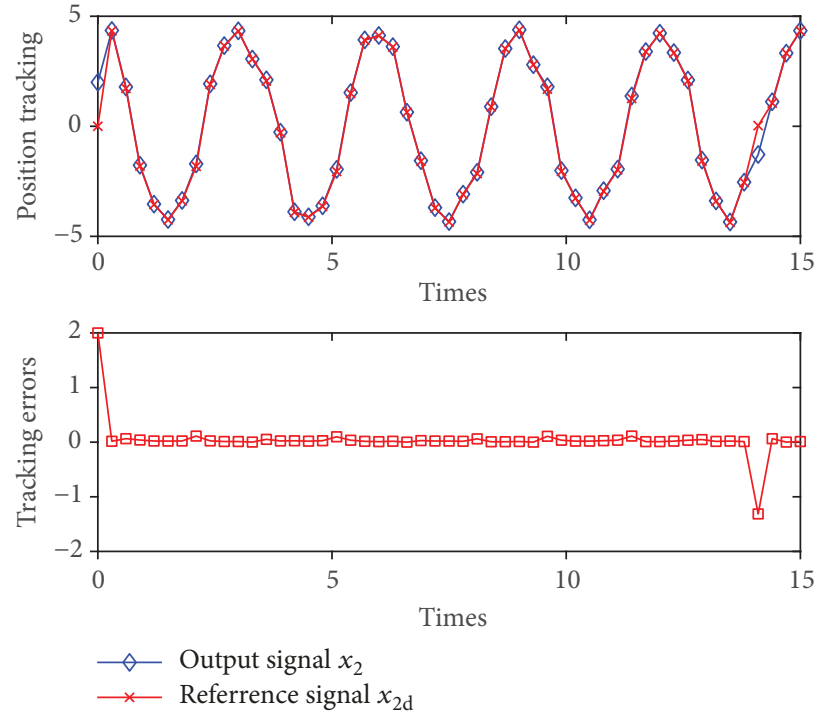

FIgURE 5: The control results and tracking error of $x_{2}$ with $y_{d}=2$ $\sin ((2 / 3) \pi t)$.
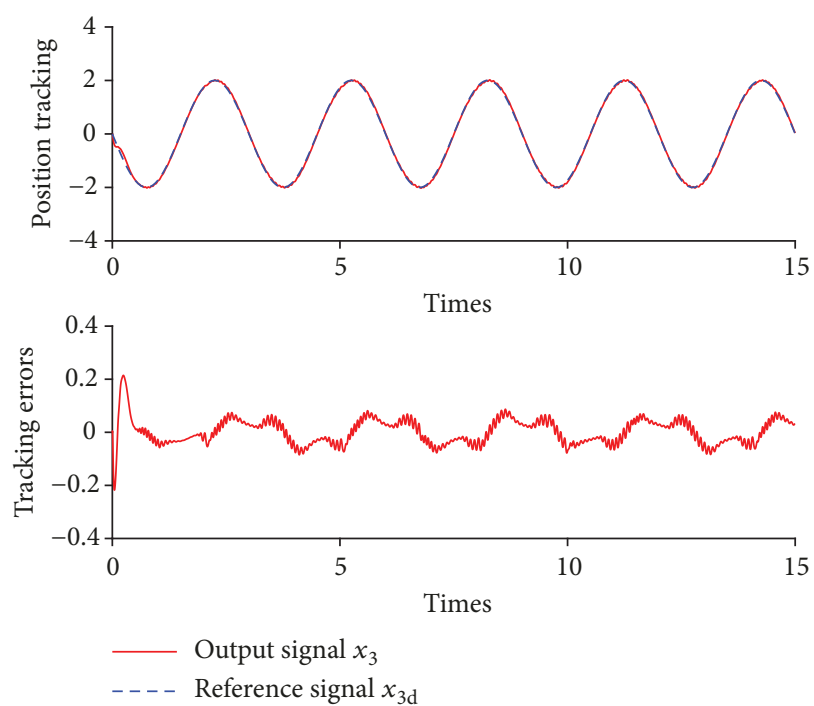

FIGURE 6: The control results and tracking error of $x_{3}$ with $y_{d}=2$ $\sin ((2 / 3) \pi t)$.

with parameters $k=6, z=3$, and $\omega=0.001$; the function is chosen as $\sigma(x)=3 /\left(1+e^{-7 x}\right)+1$ and $L=10$.

The control results and the tracking errors are expressed in Figures 4-11. It is shown that the proposed neural adaptive controller has good control precision and shortened convergence time. But the control precision is concerned with the amplitude of the reference input. Different reference input amplitude has different control precision. The control results of motor position tracking and velocity tracking are illustrated in Figures 6 and 7 with $y_{d}=2 \sin ((2 / 3) \pi t)$. From Figure 6, one can obtain that the motor has tracked the reference position within 1 second and the mean absolute error (MAE) is equal to 0.0686 . Then, the velocity of motor tracking has similar results from Figure 7. The control results of load position tracking and velocity tracking are shown in 

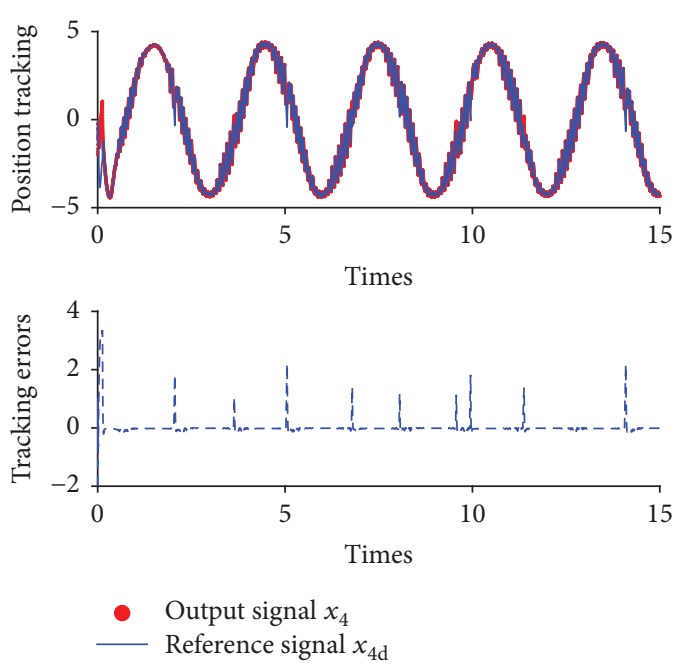

FIGURE 7: The control results and tracking error of $x_{4}$ with $y_{d}=2$ $\sin ((2 / 3) \pi t)$.
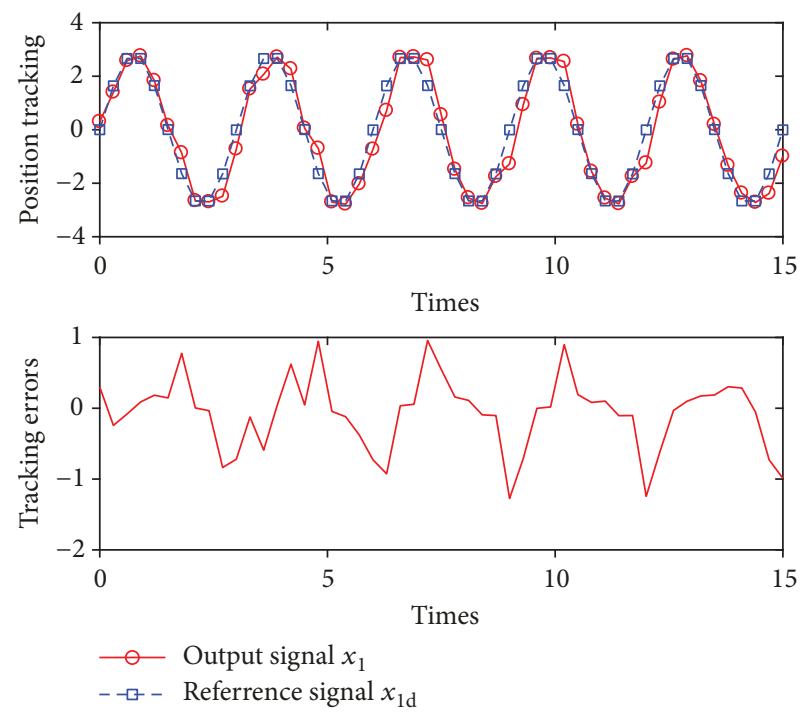

FIGURE 8: The control results and tracking error of $x_{1}$ with $y_{d}=$ $2.8 \sin ((2 / 3) \pi t)$.

Figures 4 and 5 with $y_{d}=2 \sin ((2 / 3) \pi t)$. It is illustrated that the load position can track the reference signals rapidly as well as the velocity tracking of the load.

If the input signals have larger amplitude, for example, $y_{d}=2.8 \sin ((2 / 3) \pi t)$, the control results and errors are shown in Figures 8-11. From Figures 8 and 10, the maximum errors are 0.9566 and 1.0054, respectively. But in Figures 4 and 6, the maximum errors are 0.3121 and 0.2042 , respectively. It is clearly illustrated that the amplitude of the reference input can influence the tracking precision of the adaptive control. But in velocity tracking, the accuracy of the control results are similar at a different reference input, and that can be seen from Figures 5, 7, 9, and 11. In short, the simulation results have verified the effectiveness of the proposed neural adaptive control.
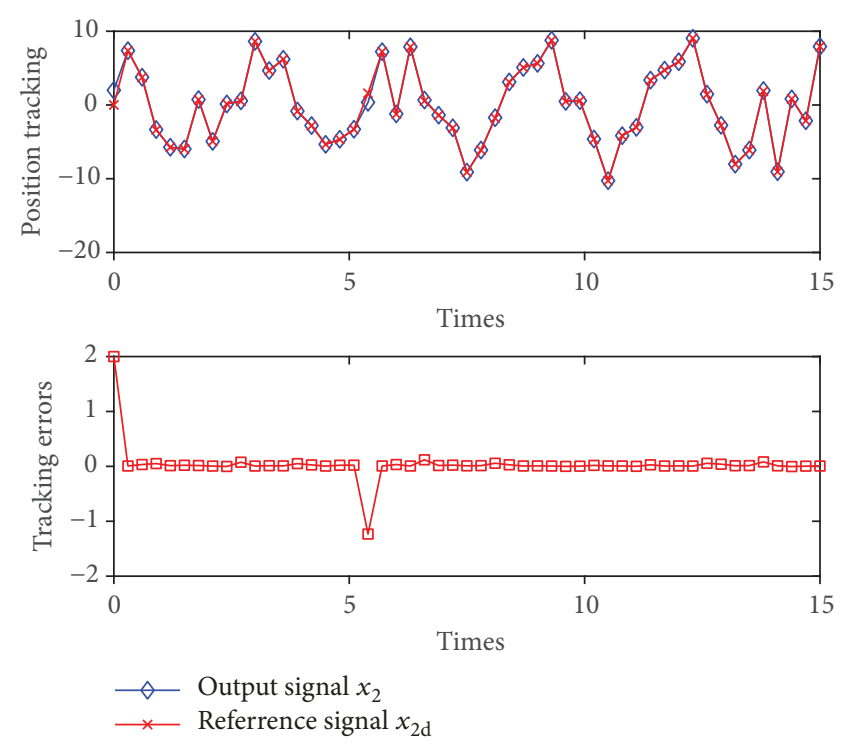

FIGURE 9: The control results and tracking error of $x_{2}$ with $y_{d}=$ $2.8 \sin ((2 / 3) \pi t)$.
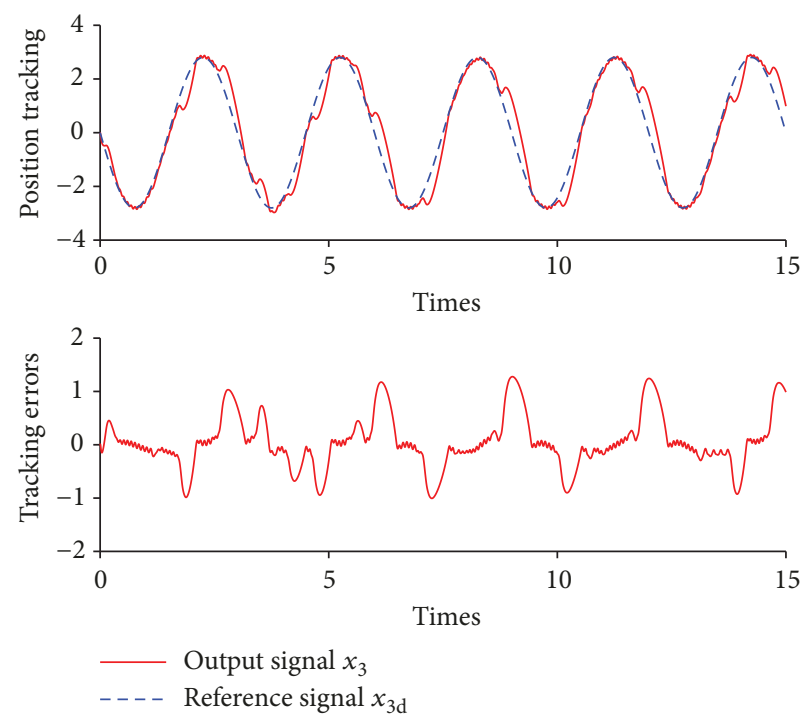

FIgURE 10: The control results and tracking error of $x_{3}$ with $y_{d}=$ $2.8 \sin ((2 / 3) \pi t)$.

\section{Conclusion}

This paper proposed an HGSO and an adaptive HONN controller for hysteresis nonlinearity of a motor driving servo system where the hysteresis nonlinearity was modeled through the Bouc-Wen model. The hysteresis motor driving servo system first has been transformed to a state space form by coordinate transformation. Then, a new HGSO was proposed, and in order to simplify the controller design, a filter was adopted for tracking errors and the adaptive HONN control was designed. Finally, a Lyapunov function candidate guaranteed all the signals of the bounded system. Simulations verified that the proposed approaches can 

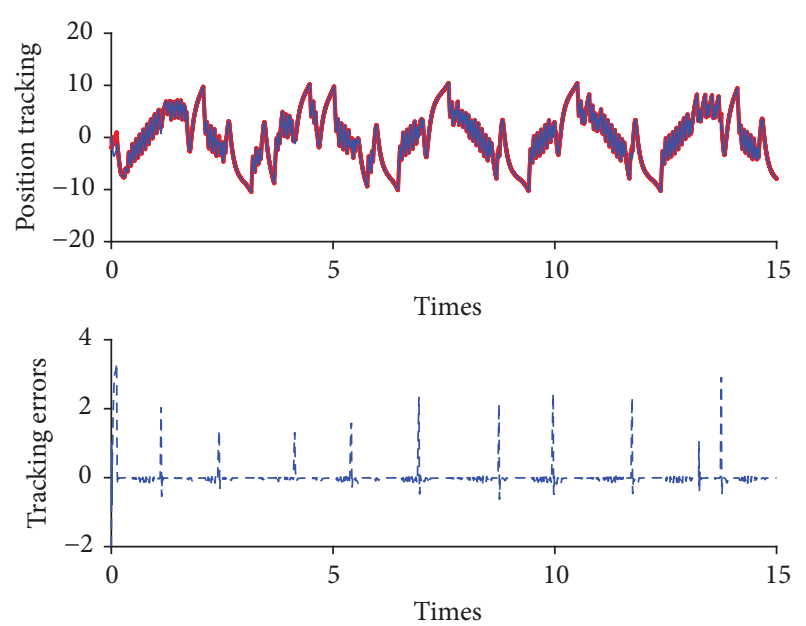

- Output signal $x_{4}$ Reference signal $x_{4 \mathrm{~d}}$

Figure 11: The control results and tracking error of $x_{4}$ with $y_{d}=$ $2.8 \sin ((2 / 3) \pi t)$.

accurately control the hysteresis nonlinearity motor driving servo system with Bouc-Wen model.

\section{Data Availability}

The data used to support the findings of this study are available from the corresponding author upon request.

\section{Conflicts of Interest}

The authors declare that there are no conflicts of interest.

\section{Acknowledgments}

This work is supported by the National Natural Science Foundation of China (61433003), Shandong Natural Science Foundation of China (ZR2017MF048), Shandong Key Research and Development Programme (2016GGX105013), Shandong Science and Technology Program of Higher Education (J17KA214), Scientific Research Foundation of Shandong University of Science and Technology for Recruited Talents (2016RCJJ035), and Tai'an Science and Technology Development Program (2017GX0017).

\section{References}

[1] J. Na, A. S. Chen, G. Herrmann, R. Burke, and C. Brace, "Vehicle engine torque estimation via unknown input observer and adaptive parameter estimation," IEEE Transactions on Vehicular Technology, vol. 67, no. 1, pp. 409-422, 2018.

[2] J. Na, Y. Huang, X. Wu, G. Gao, G. Herrmann, and J. Z. Jiang, "Active adaptive estimation and control for vehicle suspensions with prescribed performance," IEEE Transactions on Control Systems Technology, pp. 1-15, 2017.

[3] S. Krupinski, G. Allibert, M. D. Hua, and T. Hamel, "An inertial-aided homography-based visual servo control approach for (almost) fully actuated autonomous underwater vehicles," IEEE Transactions on Robotics, vol. 33, no. 5, pp. 1041-1060, 2017.

[4] J. Na, M. N. Mahyuddin, G. Herrmann, X. Ren, and P. Barber, "Robust adaptive finite-time parameter estimation and control for robotic systems," International Journal of Robust and Nonlinear Control, vol. 25, no. 16, pp. 3045-3071, 2015.

[5] G. Sun, X. Ren, and D. Li, "Neural active disturbance rejection output control of multimotor servomechanism," IEEE Transactions on Control Systems Technology, vol. 23, no. 2, pp. 746-753, 2015.

[6] A. Suarez, G. Heredia, and A. Ollero, "Physical-virtual impedance control in ultralightweight and compliant dual-arm aerial manipulators," IEEE Robotics and Automation Letters, vol. 3, no. 3, pp. 2553-2560, 2018.

[7] S. Wang, X. Ren, J. Na, and X. Gao, "Robust tracking and vibration suppression for nonlinear two-inertia system via modified dynamic surface control with error constraint," Neurocomputing, vol. 203, pp. 73-85, 2016.

[8] Q. Chen, X. Ren, J. Na, and D. Zheng, "Adaptive robust finitetime neural control of uncertain PMSM servo system with nonlinear dead zone," Neural Computing and Applications, vol. 28, no. 12, pp. 3725-3736, 2017.

[9] W. Zhao, X. Ren, and S. Wang, "Parameter estimation-based time-varying sliding mode control for multimotor driving servo systems," IEEE/ASME Transactions on Mechatronics, vol. 22, no. 5, pp. 2330-2341, 2017.

[10] Y. Wang, Z. Zhu, and Z. Zuo, "A novel design method for resolver-to-digital conversion," IEEE Transactions on Industrial Electronics, vol. 62, no. 6, pp. 3724-3731, 2015.

[11] J. Na, Q. Chen, X. Ren, and Y. Guo, “Adaptive prescribed performance motion control of servo mechanisms with friction compensation," IEEE Transactions on Industrial Electronics, vol. 61, no. 1, pp. 486-494, 2014.

[12] J. Na, G. Herrmann, and K. Zhang, "Improving transient performance of adaptive control via a modified reference model and novel adaptation," International Journal of Robust and Nonlinear Control, vol. 27, no. 8, pp. 1351-1372, 2017.

[13] S. Wang, X. Ren, J. Na, and T. Zeng, "Extended-state-observerbased funnel control for nonlinear servomechanisms with prescribed tracking performance," IEEE Transactions on Automation Science and Engineering, vol. 14, no. 1, pp. 98108, 2017.

[14] S. Wang, J. Na, and X. Ren, "Rise-based asymptotic prescribed performance tracking control of nonlinear servo mechanisms," IEEE Transactions on Systems, Man, and Cybernetics: Systems, pp. 1-12, 2017.

[15] S. Wang, X. Ren, and J. Na, "Adaptive dynamic surface control based on fuzzy disturbance observer for drive system with elastic coupling," Journal of the Franklin Institute, vol. 353, no. 8, pp. 1899-1919, 2016.

[16] D. Zhang, M. Jia, Y. Liu, Z. Ren, and C. S. Koh, "Comprehensive improvement of temperature-dependent Jiles-Atherton model utilizing variable model parameters," IEEE Transactions on Magnetics, vol. 54, no. 3, pp. 1-4, 2018.

[17] L. Perkkio, B. Upadhaya, A. Hannukainen, and P. Rasilo, "Stable adaptive method to solve FEM coupled with JilesAtherton hysteresis model," IEEE Transactions on Magnetics, vol. 54, no. 2, pp. 1-8, 2018.

[18] M. A. Zaman and M. A. Matin, "Optimization of Jiles-Atherton hysteresis model parameters using Taguchi's method," IEEE Transactions on Magnetics, vol. 51, no. 5, pp. 1-4, 2015. 
[19] X. Gao, C. Zhang, C. Zhu, and X. Ren, "Identification and control for Hammerstein systems with hysteresis non-linearity," IET Control Theory \& Applications, vol. 9, no. 13, pp. 19351947, 2015.

[20] Z. Li, X. Zhang, C. Y. Su, and T. Chai, "Nonlinear control of systems preceded by Preisach hysteresis description: a prescribed adaptive control approach," IEEE Transactions on Control Systems Technology, vol. 24, no. 2, pp. 451460, 2016.

[21] Z. Li, J. Shan, and U. Gabbert, "Development of reduced Preisach model using discrete empirical interpolation method," IEEE Transactions on Industrial Electronics, vol. 65, no. 10, pp. 8072-8079, 2018.

[22] I. Ahmad, "Two degree-of-freedom robust digital controller design with Bouc-Wen hysteresis compensator for piezoelectric positioning stage," IEEE Access, vol. 6, pp. 17275-17283, 2018.

[23] R. Xu and M. Zhou, "Sliding mode control with sigmoid function for the motion tracking control of the piezo-actuated stages," Electronics Letters, vol. 53, no. 2, pp. 75-77, 2017.

[24] G. Xuehui and S. Bo, "Identification for Bouc-Wen hysteresis system with Hopfield neural network," in 2017 9th International Conference on Modelling, Identification and Control (ICMIC), pp. 248-253, Kunming, China, July 2017.

[25] X.-H. Gao, X.-M. Ren, F. Zheng, and Q.-Z. Wang, "Prescribed performance adaptive control for hysteresis Hammerstein system," Beijing Ligong Daxue Xuebao/Transaction of Beijing Institute of Technology, vol. 36, no. 4, pp. 412-416, 2016.

[26] X. Zhang, Z. Xu, C. Y. Su et al., "Fuzzy approximator based adaptive dynamic surface control for unknown time delay nonlinear systems with input asymmetric hysteresis nonlinearities," IEEE Transactions on Systems, Man, and Cybernetics: Systems, vol. 47, no. 8, pp. 2218-2232, 2017.

[27] G. Wang, G. Chen, H. Zhou, and F. Bai, "Modeling and tracking control for piezoelectric actuator based on a new asymmetric hysteresis model," IEEE/CAA Journal of Automatica Sinica, vol. 4, no. 4, pp. 782-791, 2017.

[28] R. Bouc, "Modle mathmatique d'hystrsis: application aux systmes un degr de libert," Acustica, vol. 24, pp. 16-25, 1971.

[29] Y. K. Wen, "Method for random vibration of hysteretic systems," Journal of Engineering Mechanics, vol. 102, no. 2, pp. 249-263, 1976.

[30] S. S. Ge, J. Zhang, and T. H. Lee, "Adaptive neural network control for a class of MIMO nonlinear systems with disturbances in discrete-time," IEEE Transactions on Systems, Man, and Cybernetics, Part B (Cybernetics), vol. 34, no. 4, pp. 1630-1645, 2004.

[31] Q. Chen, L. Shi, J. Na, X. Ren, and Y. Nan, “Adaptive echo state network control for a class of pure-feedback systems with input and output constraints," Neurocomputing, vol. 275, pp. 1370-1382, 2018.

[32] D. Zheng, W. F. Xie, X. Ren, and J. Na, "Identification and control for singularly perturbed systems using multitime-scale neural networks," IEEE Transactions on Neural Networks and Learning Systems, vol. 28, no. 2, pp. 321-333, 2017.

[33] A. Y. Alanis, E. N. Sanchez, and A. G. Loukianov, "Discretetime adaptive backstepping nonlinear control via high-order neural networks," IEEE Transactions on Neural Networks, vol. 18, no. 4, pp. 1185-1195, 2007.

[34] W. Zhao, X. Ren, and X. Gao, "Synchronization and tracking control for multi-motor driving servo systems with backlash and friction," International Journal of Robust and Nonlinear Control, vol. 26, no. 13, pp. 2745-2766, 2016.

[35] J. Na, X. Ren, and D. Zheng, "Adaptive control for nonlinear pure-feedback systems with high-order sliding mode observer," IEEE Transactions on Neural Networks and Learning Systems, vol. 24, no. 3, pp. 370-382, 2013.

[36] A. Zou, K. Kumar, and Z. Hou, "Quaternion-based adaptive output feedback attitude control of spacecraft using Chebyshev neural networks," IEEE Transactions on Neural Networks, vol. 21, no. 9, pp. 1457-1471, 2010. 


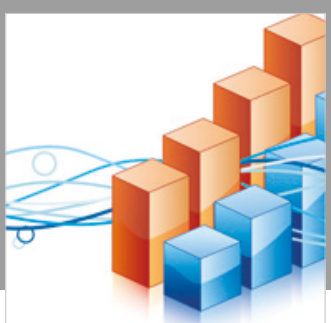

Advances in

Operations Research

\section{-n-m}
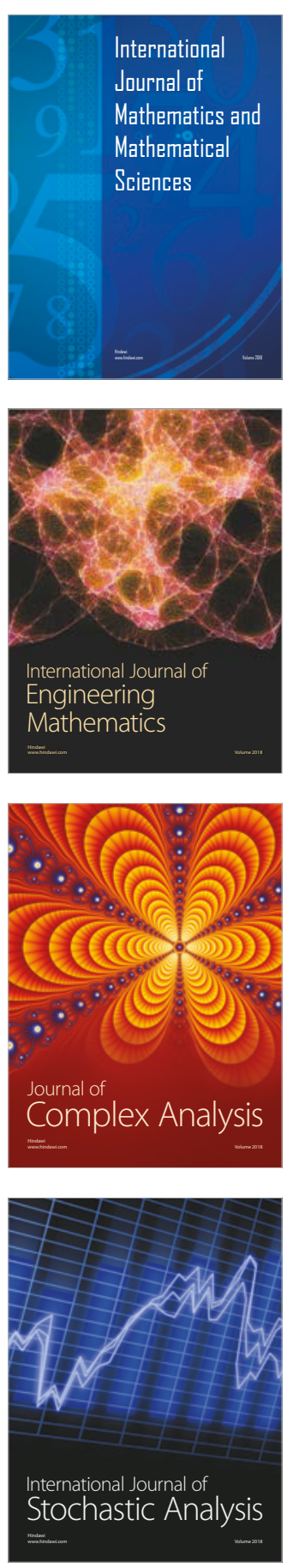
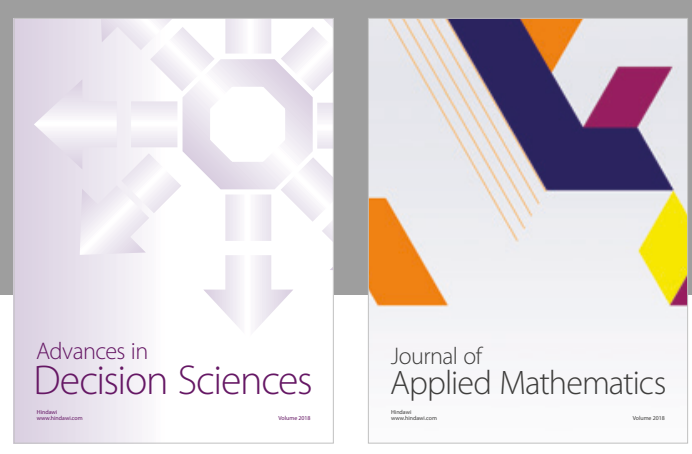

Journal of

Applied Mathematics
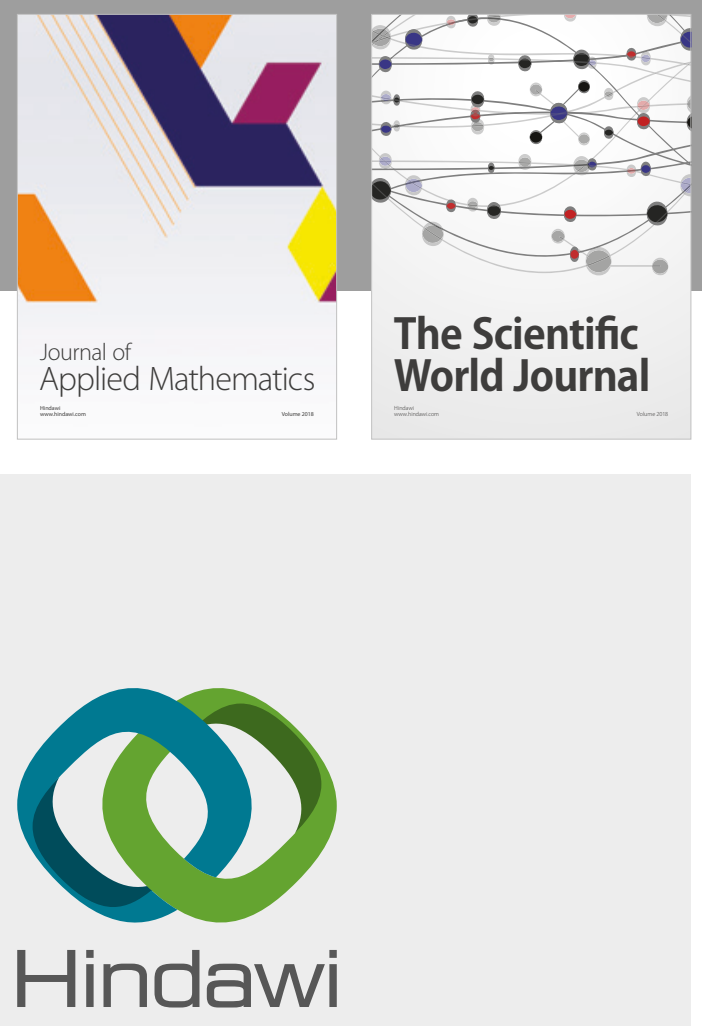

Submit your manuscripts at

www.hindawi.com

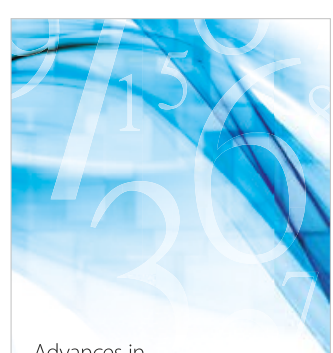

Advances in
Numerical Analysis
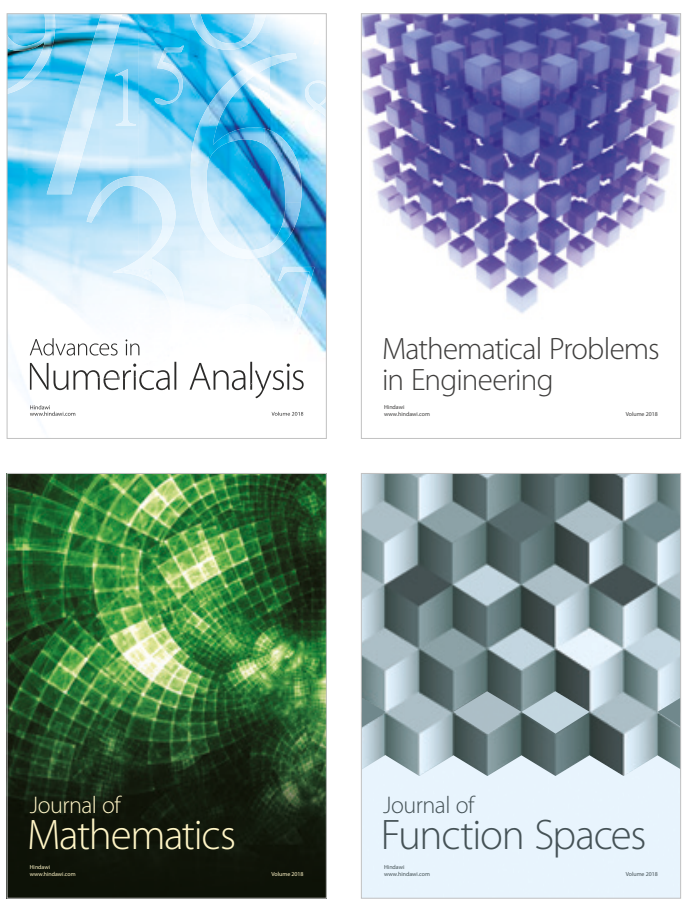

Mathematical Problems in Engineering

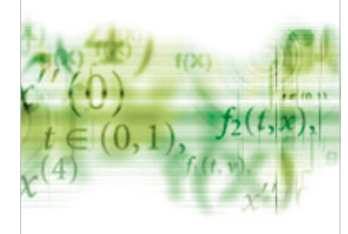

International Journal of

Differential Equations

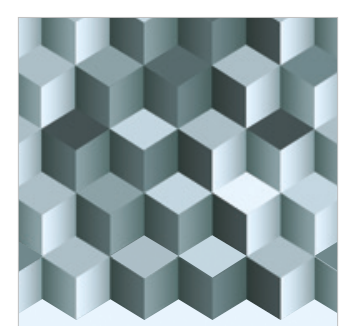

Journal of

Function Spaces
The Scientific

World Journal

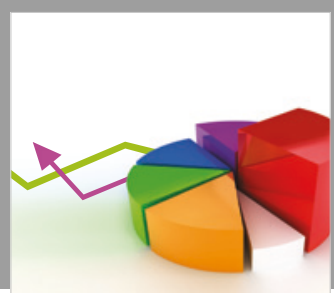

Journal of

Probability and Statistics
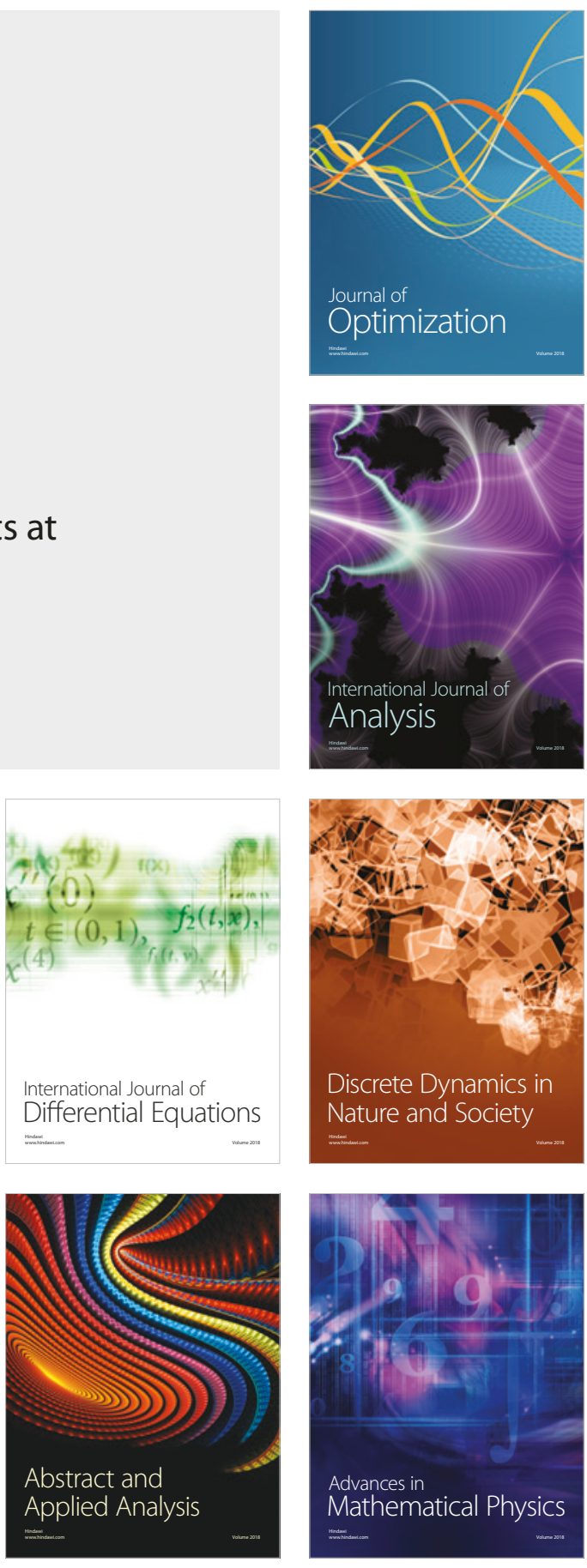